\title{
Cancer Patient Education Network
}

National Cancer Institute

\section{Source}

National Cancer Institute. Cancer Patient Education Network. NCI Thesaurus. Code C39345.

The Cancer Patient Education Network is an $\mathrm{NCl}$-sponsored org anization of cancer patient education leaders from $\mathrm{NCl}$-designated comprehensive and clinical centers nationwide. 